\title{
Peripartum Length of Stay for Women with Depressive Symptoms during Pregnancy
}

\author{
Christie A. Lancaster, M.D.,', Heather A. Flynn, Ph.D., ${ }^{1}$ Timothy R.B. Johnson, M.D., \\ Sheila M. Marcus, M.D., ${ }^{3}$ and Matthew M. Davis, M.D., MAPP $2,4,5$
}

\begin{abstract}
Background: Approximately 1 in 10 women suffers from depression during pregnancy. Little is known about whether antepartum depression affects a mother's length of stay at delivery. We aimed to compare peripartum length of stay in women with and without depressive symptoms during pregnancy.

Methods: This study involved secondary data analysis of a larger study exploring antepartum depression. Each subject completed the Center for Epidemiological Studies Depression Scale (CES-D). We used bivariate analyses to compare patient characteristics of women with and without an elevated CES-D, and we used a multivariate Poisson regression to evaluate predictors of length of stay.

Results: The study sample included 867 pregnant women. Overall, $18 \%$ of study subjects scored $\geq 16$ on the CESD. In bivariate analyses, a longer stay was associated with an elevated CES-D and minority race, antepartum complications, cesarean delivery, prematurity, multiple gestation, and neonatal length of stay. In the final multivariate model adjusting for sociodemographic, antepartum, and obstetric factors, an elevated CES-D was associated with a significantly longer peripartum stay (0.26 days, CI 0.04-0.48).
\end{abstract}

Conclusions: Depressive symptoms during pregnancy predict an increase in peripartum length of stay.

\section{Introduction}

A NTEPARTUM DEPRESSION affects approximately 1 in 10 women at various points during pregnancy. ${ }^{1}$ Although depression has been variably associated with hospital length of stay in clinical populations outside of women in the puerperium, $^{2-9}$ little is known about whether antepartum depression affects the maternal length of stay at delivery (hereafter called peripartum stay). Any relationship between antepartum depression and peripartum length of stay is clinically meaningful because perinatal depression is common and childbirth is the second leading cause of hospitalization in the United States. ${ }^{10}$

We hypothesized that depression may affect peripartum stay through several potential mechanisms. First, depression is linked to adverse obstetric outcomes, including preterm delivery and preeclampsia ${ }^{11-13}$ and, therefore, could lengthen maternal stay by increasing the frequency of such complications. Second, we hypothesized that depressed mothers taking antidepressants could stay longer as a result of lengthened neonatal observations because use of selective serotonin reuptake inhibitors (SSRIs) is associated with a neonatal withdrawal syndrome. ${ }^{14-18}$ Finally, depression may not independently influence peripartum stay, but it could appear to do so through an association with socioeconomic status (SES) or substance abuse.

The primary objective of this study was to compare peripartum length of stay, the duration of time between admission to the obstetric unit and postpartum discharge, in women with vs. women without depressive symptoms during pregnancy. In addition, we aimed to determine if any relationship between depression and peripartum stay was explained by increased obstetric complications, longer neonatal observation, or associations with SES or maternal substance use. We also compared mean hospital charges for the peripartum stay in women with vs. without an elevated depression screen.

\section{Materials and Methods}

\section{Recruitment and study sample}

This study involved secondary data analysis of the Health and Pregnancy Project (HPP), a cohort study examining general health behaviors, substance abuse, and depression in pregnancy conducted from 1999 through 2003 by two of the

\footnotetext{
${ }^{1}$ Department of Obstetrics and Gynecology, ${ }^{2}$ Division of General Internal Medicine, ${ }^{3}$ Department of Psychiatry, Women's Mood Disorders Program, ${ }^{4}$ Child Health Evaluation and Research (CHEAR) Unit, Division of General Pediatrics, and ${ }^{5}$ Gerald R. Ford School of Public Policy, University of Michigan, Ann Arbor, Michigan.
} 
investigators (S.M.M. and H.A.F.). A convenience sample of pregnant women was recruited from a group of obstetrics clinics throughout southeastern Michigan. Each subject completed a self-administered survey while waiting for her prenatal visit. All pregnant women were approached by research staff and asked to participate, and $90 \%$ of all women approached agreed to complete the screening survey. Women who chose not to participate refused further contact with the research assistant. Therefore, it was not possible to collect information on their characteristics. Comprehensive written consent was obtained from each study participant.

During this time period, 1331 women were screened. For the current study, we included HPP subjects who were patients in the investigators' hospital system in order to link to delivery information via the medical record. These 929 women comprised the study sample. We excluded women with fetal deaths in utero $(n=15)$ and women who delivered at $<20$ weeks gestational age $(n=9)$. Of the remaining 914 women who met study inclusion criteria, $47(5.1 \%)$ had missing delivery data, with a presumed delivery in another healthcare system. Thus, the final sample included 867 women. Women entered the study at various points in gestation, including all three trimesters; the mean gestational age study participation was 25.8 weeks (SD 9.6, median 27, IQR 18-34).

The hospital system uses a competency-based model for pregnancy care that requires that competency in self-care and infant care is validated before the postpartum discharge. ${ }^{19}$ The University of Michigan Medical School Institutional Review Board approved all procedures for the HPP and for this study.

\section{Survey instrument and clinical data}

General survey measures included demographic information, ratings of overall health, and use of prescription medications. In addition, past history of depression was measured using items derived from the Diagnostic Interview Schedule (DIS-III-R). ${ }^{20}$ These items ask participants if " . . you had two weeks or more when nearly every day you felt sad, blue, or depressed or in which you lost all interest in things like work?" The sensitivity of these items as screeners for depression ranges from 0.83 to $0.94 .^{21}$

Current depressive symptomatology was measured by the Center for Epidemiological Studies Depression Scale (CESD). ${ }^{22}$ Items on the CES-D cover the previous 7 days and are rated on a 4-point scale. A total score is derived by summing the ratings across the scale's 20 items. The CES-D shows good internal consistency in the general population (Cronbach's alpha $=0.84) .{ }^{23}$ In a postpartum sample, the sensitivity and specificity of the CES-D to detect depression (major and minor) were 0.60 and 0.92 , respectively. ${ }^{24}$ This is comparable to the diagnostic properties of other depression screening instruments in the postpartum population. ${ }^{1} \mathrm{We}$ used the standard cutoff point of $\geq 16$ to determine elevated depressive symptoms. ${ }^{25,26}$ Additional questions evaluated the use of depression medications in the 2 years before the study. The survey also assessed if women were receiving counseling, psychotherapy, or medication for emotional problems at the time of screening.

Problem alcohol use was assessed with the TWEAK alcohol screener. The TWEAK is a 5-item screener that contains questions from the MAST, CAGE, and T-ACE. ${ }^{27}$ It yields a total severity score of $0-7$. Using a cutoff score of 2 , the TWEAK demonstrates good sensitivity and specificity in screening for risk drinking in pregnant women. ${ }^{28}$ In the HPP study, women with a positive alcohol screener or an elevated CES-D received psychoeducational materials in the mail.

For each woman in the sample, we used hospital claims data to collect discharge diagnoses, charges, procedures, and length of stay at delivery. Peripartum length of stay was recorded in the number of whole days from the date of admission to the obstetric unit to the date of postpartum discharge. In addition, we reviewed the electronic medical record to extract breast vs. bottle feeding status and obstetric outcomes, including antepartum and postpartum complications. We calculated a modified Kotelchuck Adequacy of Prenatal Care Utilization Index. ${ }^{29}$ The Kotelchuck Index uses two data points to categorize the adequacy of prenatal care. First, the time of initiation of prenatal care is recorded. Second, the number of prenatal care visits attended is compared with the expected number of visits for the period between the initiation of care and delivery, based on prenatal care standards of the American College of Obstetricians and Gynecologists (ACOG). Finally, the index combines these two data points to create a single summary score of adequate plus, adequate, intermediate, or inadequate. Because some of our study subjects were referred in for care, we did not have complete data for the timing of the first prenatal visit. Therefore, we calculated prenatal care adequacy based on the number of weeks from the time of entry into the study health system. We categorized women by the percentage of recommended visits attended during this interval, using the cutoffs of the original Kotelchuck Index (inadequate, $<50 \%$ of expected visits; intermediate, $50 \%-79 \%$; adequate, $80 \%-109 \%$; adequate plus, $>109 \%$ ). We also obtained data from accompanying demographic records regarding insurance status of the mother and the child.

\section{Statistical analysis}

We used descriptive statistics to report patient characteristics and obstetric outcomes for the study population. We used $t$ tests and chi-square analyses to compare sociodemographic factors, substance abuse, and obstetric outcomes between women with and without depressive symptoms in pregnancy, and we used a $t$ test for samples with unequal variances to compare hospital charges between these same subgroups.

The primary outcome of interest for this project was peripartum length of stay. We used nonparametric statistics because of the skewed distribution of this outcome, and we fit a Poisson regression to the data because length of stay was recorded in counts of whole days. We reviewed the literature on peripartum length of stay to determine the independent variables for inclusion (Table 1). We examined the bivariate Poisson association of each of these variables with the primary outcome, and variables with an association with $p<0.2$ were included in the multivariate Poisson regression. We examined the change in regression coefficients after adding each predictor to the model. Using the final Poisson regression, we calculated marginal effects for each independent variable's association with peripartum length of stay, using robust confidence intervals (CI) for each of the associations. Finally, we tested our model for goodness of fit using the Pearson 
Table 1. Predictor Variables

Neonatal factors
Gestational age at delivery
Neonatal length of stay
Obstetric factors
Adequacy of prenatal care, modified Kotelchuck Index
Antepartum complications
Postpartum complications
Mode of delivery
Feeding status, breast vs. bottle feeding
Parity
Multiple pregnancy
Sociodemographic factors
Maternal age
Education
Race
Insurance status
Marital status
Employment
Psychosocial factors
Smoking Status
TWEAK $\geq 2$
CESD $\geq 16$
Treatment status for depression

statistic ( $p=1$ representing an appropriate fit). All analyses were conducted with STATA ${ }^{\circledR} 10$ (StataCorp, College Station, TX).

\section{Results}

The final sample contained 867 women with complete delivery data. Women with missing delivery data $(n=47)$ were younger, less educated, and more likely to be single and to have public insurance. They did not differ by race, employment, or parity from those who remained in the study. Women with missing delivery data were more likely than those with complete data to have an elevated depression screen; $34 \%$ scored $\geq 16$ on the CES-D (vs. $18.9 \%$ ) (chisquare $=6.5, p=0.01$ ).

In addition, of the 867 women with complete delivery data, 23 women did not complete the CES-D and 42 women did not complete the TWEAK. Therefore, the final regression model contained 825 women, representing $95.1 \%$ of the total number of women with complete delivery data. We ran the regression model with and without imputed data for the TWEAK, and the results were not significantly different. For the rest of this article, we present results using the nonimputed data.

\section{Sample characteristics}

Participant characteristics are shown in Table 2. The average maternal age at study entry was 29.4 years (SD 5.5, median 29, IQR 18-33). Most women were Caucasian, and the majority had private health insurance. Almost $90 \%$ of the women were married or cohabiting. Over one half of the women in the study graduated from college. More women reported alcohol use $(15.3 \%)$ than cigarette smoking $(13.0 \%)$ during pregnancy.

The mean gestational age at study participation was 25.8 weeks (SD 9.6). Most mothers rated adequate/adequate plus on a modified Kotelchuck Index. Over $25 \%$ of women experienced at least one antepartum complication; the most com- mon complications were hypertensive disorders, gestational diabetes, and preterm premature rupture of membranes (PPROM).

Delivery outcomes are displayed in Table 3. The mean gestational age at delivery was 38.8 weeks (SD 2.4); $27.8 \%$ $(n=241)$ of women received a cesarean delivery, and $41.9 \%$ of these 241 deliveries were scheduled, elective cesareans $(58.1 \%$ were indicated cesarean deliveries after admission). Only $4.6 \%$ of mothers experienced a postpartum complication. The majority of mothers breastfed their infants.

Overall, $18.3 \%$ of mothers scored above the cutoff for depressive symptomatology on the CES-D $(n=159) ; 20 \%$ of the women with a positive CES-D reported any current form of treatment $(n=30)$, and over one half of the women with a positive CES-D had a prior history of depression in their lifetime $(n=96)$. In addition, $3.4 \%$ of women with a CES-D $<16$ reported current treatment for depression, likely reflecting women who had improved symptomatology on their current treatment regimen.

In bivariate analyses, depressed mothers were younger and less educated. They were more likely to be single, unemployed, and publicly insured (Table 2). One quarter of depressed mothers reported smoking during the index pregnancy, and they were twice as likely to have an elevated TWEAK. Depressed women did not differ by frequency of obstetric complications or mode of delivery, including the type of Cesarean delivery (scheduled, elective vs. for obstetric indications after admission) (Tables 2 and 3). In addition, of women with a history of depression in the 2 years before pregnancy $(n=72)$, less than half $(n=30,45.4 \%)$ reported any antidepressant use during the past 2 years.

In our study population, the mean hospital charges for a maternal peripartum stay equaled $\$ 5689$ (SD $\pm \$ 2267$ ). The mean charges were about $10 \%$ higher in women with a positive CES-D (\$542, $p=0.05)$.

\section{Peripartum length of stay and antepartum depression}

The mean peripartum length of stay was 2.1 days (SD \pm 1.3 , median 2, IQR 1-2). In bivariate analyses, antepartum depressive symptoms were associated with significantly longer peripartum stay (0.36 days). In addition, a longer stay was significantly associated with the following variables: lower educational attainment, minority race, number of antepartum complications, cesarean delivery, prematurity, multiple gestation, and neonatal length of stay (Table 4). Multiparous women had shorter lengths of stay, and breastfeeding women showed a trend toward shorter stays, although this did not reach statistical significance $(p=0.06)$. Women with inadequate prenatal care also spent fewer days in the hospital. The maternal length of stay was not associated with smoking, insurance status, employment, marital status, maternal age, or frequency of postpartum complications. We saw a trend toward longer stays in women with an elevated TWEAK, but this relationship did not reach statistical significance $(p=0.10)$.

In the final multivariate model, longer peripartum stays were significantly associated with antepartum depressive symptoms and with minority race, antepartum complications, cesarean delivery, and a longer neonatal hospital stay (Table 4). A shorter stay was associated with multiparity. The association between antepartum depression and peripartum 
TABle 2. Subject Characteristics ${ }^{\mathrm{a}}$

\begin{tabular}{|c|c|c|c|c|}
\hline & Total $^{\mathrm{b}}$ & $C E S-D \geq 16^{\mathrm{a}}$ & $C E S-D<16^{\mathrm{C}}$ & $\mathrm{p}$ \\
\hline Age & $29.4(5.5)$ & $28.1(5.6)$ & $29.7(5.5)$ & $<0.01$ \\
\hline \multicolumn{5}{|l|}{ Race } \\
\hline Non-Hispanic white & $665(76.7)$ & $117(74.0)$ & $533(77.8)$ & 0.31 \\
\hline Married/cohabiting & $779(89.8)$ & $121(77.1)$ & 639 (93.7) & $<0.01$ \\
\hline \multicolumn{5}{|l|}{ Education } \\
\hline$\leq$ High School & $196(22.6)$ & $57(35.8)$ & $130(19.0)$ & \multirow[t]{3}{*}{$<0.01$} \\
\hline Some college/college graduate & $407(46.9)$ & $74(46.5)$ & $320(46.7)$ & \\
\hline Beyond college & $264(30.4)$ & $28(17.6)$ & $235(34.3)$ & \\
\hline \multicolumn{5}{|l|}{ Employment } \\
\hline Unemployed & $334(38.5)$ & $81(51.3)$ & $243(35.5)$ & \multirow[t]{3}{*}{$<0.01$} \\
\hline Part-time & $153(17.6)$ & $24(15.2)$ & $127(18.6)$ & \\
\hline Full-time & $377(43.5)$ & $53(33.5)$ & $314(45.9)$ & \\
\hline \multicolumn{5}{|l|}{ Insurance status } \\
\hline Public (Medicare/Medicaid) & $60(6.9)$ & $24(15.1)$ & $31(4.5)$ & \multirow[t]{2}{*}{$<0.01$} \\
\hline Private & $805(92.8)$ & $135(84.9)$ & $652(95.5)$ & \\
\hline Multiparous & $462(53.3)$ & $91(57.2)$ & $355(51.8)$ & 0.22 \\
\hline Smoker & $113(13.0)$ & $41(25.9)$ & $66(9.7)$ & $<0.01$ \\
\hline Alcohol use in index pregnancy & $133(15.3)$ & $28(17.6)$ & $103(15.1)$ & 0.42 \\
\hline TWEAK $\geq 2$ & $78(9.0)$ & $24(16.0)$ & $53(8.1)$ & $<0.01$ \\
\hline Lifetime history of depression & $271(31.3)$ & $96(62.3)$ & $167(24.5)$ & $<0.01$ \\
\hline Current depression treatment & $56(6.5)$ & $30(20.0)$ & $21(3.4)$ & $<0.01$ \\
\hline \multicolumn{5}{|l|}{ Kotelchuck Index, modified } \\
\hline Inadequate & $103(11.9)$ & $18(11.3)$ & $80(11.7)$ & \multirow[t]{3}{*}{0.37} \\
\hline Intermediate & $117(13.5)$ & $16(10.1)$ & $97(14.2)$ & \\
\hline Adequate/adequate plus & $647(74.6)$ & $125(78.6)$ & $508(74.2)$ & \\
\hline Antepartum complications ${ }^{\mathrm{d}}$ & $251(28.9)$ & $51(32.1)$ & $193(28.2)$ & 0.33 \\
\hline Multiple gestation & $49(5.6)$ & $9(5.7)$ & $35(5.1)$ & 0.78 \\
\hline
\end{tabular}

${ }^{\mathrm{a}}$ Age presented as mean (SD); all others presented as $n(\%)$.

${ }^{\mathrm{b}}$ Missing data (n): race (1); marital status (5); employment (3); insurance status (2); smoker (7); alcohol use (1); TWEAK (42); lifetime depression (9); current treatment (73).

${ }^{c} n=844$ with completed CES-D.

${ }^{\mathrm{d}}$ Antepartum complications, $n$ (\%): hypertensive disorders 42 (4.8); gestational diabetes 29 (3.3); autoimmune disorders 12 (1.4); IUGR 7 (0.8); fetal anomalies 13 (1.5); incompetent cervix 3 (0.3); PPROM 21 (2.4).

length of stay was partially influenced by maternal education level, mode of delivery, gestational age, and a positive TWEAK. After controlling for these factors, however, an elevated CES-D remained associated with a statistically significant increase of 0.24 days of peripartum stay.

We categorized peripartum length of stay by days, and women with a positive CES-D had a $10 \%$ higher frequency of stays of 3 days and a $30 \%$ higher frequency of stays of $\geq 4$ days (Fig. 1). Depressed women receiving current mental health treatment did not differ in length of stay from those who were not receiving treatment $(p=0.34)$. In addition, a lifetime history of depression and a history of medication use in the past 2 years were not significantly associated with peripartum length of stay.

Table 3. Delivery Outcomes by Antepartum Depression Status ${ }^{a}$

\begin{tabular}{|c|c|c|c|c|}
\hline & Total & $C E S-D \geq 16$ & $C E S-D<16$ & $\mathrm{p}$ \\
\hline Gestational age at delivery, weeks & $38.8(2.4)$ & $38.5(2.5)$ & $38.8(2.4)$ & 0.16 \\
\hline \multicolumn{5}{|l|}{ Mode of delivery, $n(\%)$} \\
\hline Spontaneous vaginal & $438(50.5)$ & $73(45.9)$ & $356(52.0)$ & 0.39 \\
\hline Assisted vaginal & $188(21.7)$ & $38(23.9)$ & $145(21.2)$ & \\
\hline Cesarean & $241(27.8)$ & $48(30.2)$ & $184(26.9)$ & \\
\hline Apgar, 1 minute & $7.9(1.5)$ & $7.8(1.5)$ & $7.9(1.5)$ & 0.65 \\
\hline Apgar, 5 minute & $8.8(0.8)$ & $8.8(0.7)$ & $8.8(0.8)$ & 0.55 \\
\hline Birth weight, $\mathrm{kg}$ & $3.3(0.6)$ & $3.3(0.7)$ & $3.4(0.6)$ & 0.27 \\
\hline Postpartum complications $n(\%)$ & $40(4.6)$ & $5(3.1)$ & $34(5.0)$ & 0.32 \\
\hline Neonatal length of stay & $3.3(8.6)$ & $3.8(7.6)$ & $3.1(8.9)$ & 0.35 \\
\hline Breastfeeding, $n(\%)$ & $674(77.7)$ & $117(74.0)$ & $543(80.4)$ & 0.07 \\
\hline Hospital charges, in \$thousands & $5.69(2.27)$ & $6.11(3.40)$ & $5.57(1.86)$ & 0.05 \\
\hline
\end{tabular}

${ }^{a}$ Missing data (n): Apgar, 1 minute (13); Apgar, 5 minute (12); birth weight (3); neonatal length of stay (3); breastfeeding (12); hospital charges (8). 
Table 4. Marginal Effect on Maternal Peripartum LENGTH OF STAY, IN DAYS

\begin{tabular}{|c|c|c|c|c|}
\hline & Unadjusted & $\mathrm{p}$ & Adjusted $^{\mathrm{a}}$ & $\mathrm{p}$ \\
\hline CES-D $\geq 16$ & 0.36 & 0.04 & 0.24 & 0.03 \\
\hline \multicolumn{5}{|l|}{ Education } \\
\hline Beyond college & Referent & & Referent & \\
\hline$\leq$ High school & 0.31 & 0.02 & 0.19 & 0.12 \\
\hline $\begin{array}{l}\text { Some college/ } \\
\text { college graduate }\end{array}$ & 0.28 & $<0.01$ & 0.04 & 0.56 \\
\hline \multicolumn{5}{|l|}{ Race } \\
\hline Non-Hispanic white & Referent & & Referent & \\
\hline Minority & 0.30 & 0.03 & 0.19 & 0.02 \\
\hline \multicolumn{5}{|l|}{ Insurance status } \\
\hline Private & Referent & & Referent & \\
\hline Public & -0.19 & 0.13 & -0.25 & 0.05 \\
\hline \multicolumn{5}{|l|}{ Kotelchuck Index } \\
\hline $\begin{array}{l}\text { Adequate/ } \\
\text { adequate plus }\end{array}$ & Referent & & Referent & \\
\hline Intermediate & -0.05 & 0.60 & 0.00 & 0.96 \\
\hline Inadequate & -0.20 & 0.03 & -0.09 & 0.22 \\
\hline $\begin{array}{l}\text { Antepartum } \\
\text { complications, sum }\end{array}$ & 0.52 & $<0.01$ & 0.36 & $<0.01$ \\
\hline \multicolumn{5}{|l|}{ Mode of delivery } \\
\hline Spontaneous vaginal & Referent & & Referent & \\
\hline Assisted vaginal & 0.21 & 0.07 & 0.05 & 0.51 \\
\hline Cesarean & 1.34 & $<0.01$ & 1.10 & $<0.01$ \\
\hline Breastfeeding & -0.26 & 0.06 & -0.06 & 0.54 \\
\hline Multiparity & -0.36 & $<0.01$ & -0.29 & $<0.01$ \\
\hline $\begin{array}{l}\text { Gestational age } \\
\text { at delivery }\end{array}$ & -0.13 & $<0.01$ & 0.02 & 0.38 \\
\hline $\begin{array}{l}\text { Neonatal length } \\
\text { of stay }\end{array}$ & 0.03 & $<0.01$ & 0.02 & $<0.01$ \\
\hline Multiple gestation & 1.21 & $<0.01$ & 0.27 & 0.19 \\
\hline TWEAK $\geq 2$ & 0.31 & 0.10 & 0.16 & 0.24 \\
\hline
\end{tabular}

${ }^{\mathrm{a}}$ Model adjusted for all variables in table.

We conducted post hoc analyses to test for interaction effects between depression and breastfeeding and depression and parity. None of the interaction terms were significantly associated with peripartum length of stay.

\section{Discussion}

The key finding of this study is that depressive symptoms during pregnancy are strongly associated with longer peripartum stay, adjusted for multiple potential confounding factors. In our sample, $18 \%$ of women had elevated depressive symptoms on an antepartum screen; on average, a positive screen increased maternal stay by about one quarter of a day. Given the mean and median lengths of stay of 2 days, this is a relative increase in length of stay of $>10 \%$. This extended length of stay was accompanied by higher hospital charges of similar magnitude.

Initially, we hypothesized that depression could influence length of peripartum stay through increased obstetric complications, longer neonatal stays, or confounding with SES or problem drinking. We controlled for each of these scenarios in our regression model. Maternal depression was partially influenced by education, problem drinking, mode of delivery, and gestational age, but after controlling for obstetric factors, demographics, treatment status, and substance abuse, antepartum depressive symptoms continued to show an independent association with length of stay at delivery. The fact

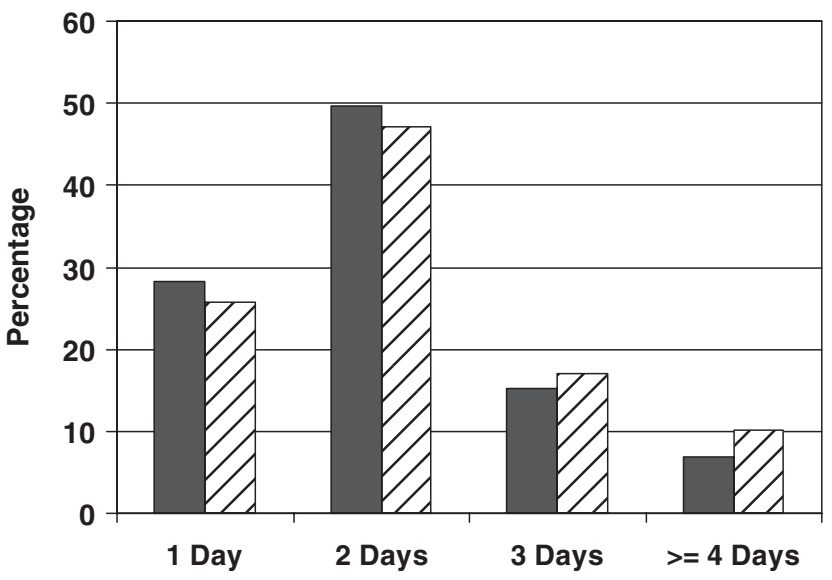

FIG. 1. Maternal length of stay by depression status. Solid bars represent percentages for subjects with CES-D $<16$. Dashed bars represent percentages for subjects with CES-D $\geq 16$.

that the depression screen score and not treatment status was associated with length of stay suggests that it is the current level of symptom severity that explains the association.

We hypothesize that this independent association may occur through several potential pathways, both before and after delivery. Depression may alter antepartum management, such as the likelihood to induce and augment labor or the type of labor anesthesia requested. Also, as depression is linked to altered neuroendocrine function, ${ }^{30,31}$ it could potentially affect labor physiology. After delivery, depression may impact postnatal transitioning, affecting one's confidence as a mother or perhaps leading to greater difficulty in initiating breastfeeding. Depression may lead to longer hospital stays also because of psychosocial interventions in the postpartum period. If a mother with depression has not received mental healthcare during her pregnancy or if her depression was previously unrecognized, providers may attempt to establish links to mental health services before discharging the mother and baby. In one study, women with psychiatric illness were more likely to be evaluated if they had a longer hospital stay at delivery. ${ }^{32}$ We will need further studies with this cohort to examine these possible associations between depression during pregnancy and peripartum stay.

Although length of stay is often used as a marker of hospital efficiency, we must interpret our findings cautiously. In other words, longer peripartum stays may use more resources but may do so wisely. Margolis et al. ${ }^{33}$ noted that the postpartum hospital stay can serve three potential public health goals: (1) "to ensure that the conditions of infants and mothers are stable," (2) "to ensure that mothers are ready to care for their new infants," and (3) "to establish linkages for ongoing care." ${ }^{\prime 33}$ Perhaps the increased stay for depressed women reflects extra time required to satisfy such goals.

Our findings contrast with those of Larsson et al., ${ }^{34}$ who did not find a significant association between maternal depressive symptoms and postpartum length of stay. There are several possible reasons for a discrepancy in our study findings. Larsson et al. screened women for depressive symptoms near term, at 35-36 weeks of gestation, rather than at 25 weeks on average as we did in this study. Furthermore, $>50 \%$ of their sample had postpartum stays of $\geq 3$ days, compared 
with a median stay of 2 days (IQR 1-2) in our study sample; this may have muted the ability to detect prolonged lengths of stay associated with antepartum depression. In addition, the subjects from that study were participants in the Swedish antenatal healthcare system, which reaches almost $100 \%$ of pregnant women in Sweden.

Our current study has several limitations. First, we relied on self-report screening measures instead of a diagnostic interview. Second, our study was cross-sectional in nature, capturing depressive symptoms at only one point in time. However, postpartum meta-analyses have shown that antepartum depression is indeed one of the strongest risk factors for subsequent postpartum depression, ${ }^{35,36}$ and a study of 360 pregnant women showed that one half of subjects with postpartum depression actually started with antepartum depression that had persisted into the postpartum period. ${ }^{37}$ Our findings may not generalize to other clinical populations, as our study population was mostly partnered, highly educated, and of high obstetric risk (over one fourth of the sample had an antepartum complication). In addition, other medical institutions may have different discharge practice patterns from those at a tertiary care, academic institution. Because of our cohort study design, we cannot ensure that we controlled for all potential confounders. Lastly, we present maternal length of stay in aggregate form, including nights spent in labor and nights after birth. This method of reporting length of stay is similar to that of other studies. ${ }^{33}$ However, we did not distinguish between effects that occurred before or after delivery. Of note, our findings also demonstrate that women with inadequate prenatal care had shorter lengths of stay. This finding has been replicated in other studies concerning peripartum length of stay. ${ }^{38,39}$ It is outside the scope of our study to explain the reasons behind this association, as we designed our study to look at depressive status and length of stay. Future studies should examine potential mechanisms that explain this association.

Prolonged maternal hospitalizations may have several potential negative effects. For the system, prolonged hospitalizations may lead to a backup of patients on the wards, which is especially cumbersome in a high turnover environment, such as that in labor and delivery. In addition, as we have shown, these prolonged hospitalizations are related to a significant increase in hospital charges. For the patient, increased time in the hospital means time away from work and family. This may also place a strain on other family members who must then take on the additional responsibilities that the mother maintained.

In summary, our findings reflect that depression is a common antepartum complication in obstetric patientsmore common than gestational diabetes or hypertensive disorders in our sample. We contribute to the existing literature on perinatal mental health by using longitudinal data to show that antepartum depressive symptoms have an independent relationship with subsequent peripartum length of stay, even after controlling for sociodemographic factors, obstetric complications, and maternal substance use. It is possible that clinical interventions for depression during the pregnancy could modify this relationship.

\section{Acknowledgments}

This work was supported by the Robert Wood Johnson Clinical Scholars Program. A poster of this work was pre- sented at the Royal College of Obstetricians and Gynaecologists 7th International Scientific Meeting, Montreal, Canada, September 17-20, 2008.

\section{Disclosure Statement}

The authors have no conflicts of interest to report.

\section{References}

1. Gaynes BN, Gavin N, Meltzer-Brody S, et al. Perinatal depression: Prevalence, screening accuracy, and screening outcomes. Evid Rep Technol Assess (Summ) 2005;119: 1-8.

2. Holmes J, House A. Psychiatric illness predicts poor outcome after surgery for hip fracture: A prospective cohort study. Psychol Med 2000;30:921-929.

3. Hosaka T, Aoki T, Watanabe T, Okuyama T, Kurosawa H. Comorbidity of depression among physically ill patients and its effect on the length of hospital stay. Psychiatry Clin Neurosci 1999;53:491-495.

4. Koopmans CT, Donker MC, Rutten FH. Length of hospital stay and health services use of medical inpatients with comorbid noncognitive mental disorders: A review of the literature. Gen Hosp Psychiatry 2005;27:44-56.

5. Ng TP, Niti M, Tan WC, Cao Z, Ong KC, Eng P. Depressive symptoms and chronic obstructive pulmonary disease: Effect on mortality, hospital readmission, symptom burden, functional status, and quality of life. Arch Intern Med 2007; 167:60-67.

6. Nuyen JJ, Spreeuwenberg PM, Groenewegen PP, van den Bos GA, Schellevis FG. Impact of preexisting depression on length of stay and discharge destination among patients hospitalized for acute stroke: Linked register-based study. Stroke 2008;39:132-138.

7. Oxlad M, Stubberfield J, Stuklis R, Edwards J, Wade TD. Psychological risk factors for increased post-operative length of hospital stay following coronary artery bypass graft surgery. J Behav Med 2006;29:179-190.

8. Saravay SSM, Lavin MM. Psychiatric comorbidity and length of stay in the general hospital. A critical review of outcome studies. Psychosomatics 1994;35:233-252.

9. Verbosky LA, Franco KN, Zrull JP. The relationship between depression and length of stay in the general hospital patient. J Clin Psychiatry 1993;54:177-181.

10. Defrances CJ, Hall MJ. 2005 National Hospital Discharge Survey. Advance data from vital and health statistics, No. 395. Hyattsville, MD: National Center for Health Statistics, 2007.

11. Kurki T, Hiilesmaa V, Raitasalo R, Mattila H, Ylikorkala O. Depression and anxiety in early pregnancy and risk for preeclampsia. Obstet Gynecol 2000;95:487-490.

12. Orr ST, James SA, Blackmore Prince C. Maternal prenatal depressive symptoms and spontaneous preterm births among African-American women in Baltimore, Maryland. Am J Epidemiol 2002;156:797-802.

13. Steer RA, Scholl TO, Hediger ML, Fischer RL. Self-reported depression and negative pregnancy outcomes. J Clin Epidemiol 1992;45:1093-1099.

14. Chambers CD, Johnson KA, Dick LM, Felix RJ, Jones KL. Birth outcomes in pregnant women taking fluoxetine. N Engl J Med 1996;335:1010-1015.

15. Costei AM, Kozer E, Ho T, Ito S, Koren G. Perinatal outcome following third trimester exposure to paroxetine. Arch Pediatr Adolesc Med 2002;156:1129-1132. 
16. Kallen B. Neonate characteristics after maternal use of antidepressants in late pregnancy. Arch Pediatr Adolesc Med 2004;158:312-316.

17. Moses-Kolko EL, Bogen D, Perel J, et al. Neonatal signs after late in utero exposure to serotonin reuptake inhibitors: Literature review and implications for clinical applications. JAMA 2005;293:2372-2383.

18. Zeskind PS, Stephens LE. Maternal selective serotonin reuptake inhibitor use during pregnancy and newborn neurobehavior. Pediatrics 2004;113:368-375.

19. Johnson TR, Zettelmaier MA, Warner PA, Hayashi RH, Avni $\mathrm{M}$, Luke B. A competency based approach to comprehensive pregnancy care. Womens Health Issues 2000;10:240-247.

20. Robins LLN, Helzer JJE, Croughan JJ, Ratcliff KKS. National Institute of Mental Health Diagnostic Interview Schedule. Its history, characteristics, and validity. Arch Gen psychiatry 1981;38:381-389.

21. Rost K, Burnam MA, Smith GR. Development of screeners for depressive disorders and substance disorder history. Med Care 1993;31:189-200.

22. Radloff LS. The CES-D scale: A self-report depression scale for research in the general population. Appl Psychol Meas 1977;1:385-401.

23. Corcoran K, Fisher J. Measures for clinical practice: A sourcebook. New York: Free Press, 1987.

24. Campbell SB, Cohn JF. Prevalence and correlates of postpartum depression in first-time mothers. I Abnorm Psychol 1991;100:594-599.

25. Husain BA, Neff JA, Harrington JB, Hughes MD, Stone RH. Depression in rural communities: Validating the CES-D scale. J Community Psychol 1980;8:20-27.

26. Myers JK, Weissman MM. Use of a self-report symptom scale to detect depression in a community sample. Am J Psychiatry 1980;137:1081-1084.

27. Chang GG. Alcohol-screening instruments for pregnant women. Alcohol Res Health 2001;25:204-209.

28. Bradley KA, Boyd-Wickizer J, Powell SH, Burman ML. Alcohol screening questionnaires in women: A critical review. JAMA 1998;280:166-171.

29. Kotelchuck M. An evaluation of the Kessner Adequacy of Prenatal Care Index and a proposed Adequacy of Prenatal Care Utilization Index. Am J Public Health 1994;84:1414-1420.
30. Field T, Hernandez-Reif M, Diego M, Figueiredo B, Schanberg S, Kuhn C. Prenatal cortisol, prematurity and low birthweight. Infant Behav Dev 2006;29:268-275.

31. Field T, Hernandez-Reif M, Diego M, Schanberg S, Kuhn C. Stability of mood states and biochemistry across pregnancy. Infant Behav Dev 2006;29:262-267.

32. Kelly R, Zatzick D, Anders T. The detection and treatment of psychiatric disorders and substance use among pregnant women cared for in obstetrics. Am J Psychiatry 2001;158: 213-219.

33. Margolis LH, Kotelchuck M, Chang HY. Factors associated with early maternal postpartum discharge from the hospital. Arch Pediatr Adolesc Med 1997;151:466-472.

34. Larsson C, Sydsjo G, Josefsson A. Health, sociodemographic data, and pregnancy outcome in women with antepartum depressive symptoms. Obstet Gynecol 2004;104:459-466.

35. Beck CT. Predictors of postpartum depression: An update. Nurs Res 2001;50:275-285.

36. Robertson E, Grace S, Wallington T, Stewart DE. Antenatal risk factors for postpartum depression: A synthesis of recent literature. Gen Hosp Psychiatry 2004;26:289-295.

37. Gotlib IH, Whiffen VE, Mount JH, Milne K, Cordy NI. Prevalence rates and demographic characteristics associated with depression in pregnancy and the postpartum. I Consult Clin Psychol 1989;57:269-274.

38. Margolis LH, Kotelchuck M. Midwives, physicians, and the timing of maternal postpartum discharge. I Nurse Midwifery 1996;41:29-35.

39. Weiss M, Ryan P, Lokken L, Nelson M. Length of stay after vaginal birth: Sociodemographic and readiness-fordischarge factors. Birth 2004;31:93-101.

Address correspondence to:

Christie A. Lancaster, M.D.

Robert Wood Johnson Clinical Scholars Program

University of Michigan

6312 Medical Science Building I

1150 West Medical Center Drive

Ann Arbor, MI 48109-5604

E-mail: chrlanca@umich.edu 
